\title{
Will There Be Any Neat Solutions to Small Problems in Cognitive Science?
}

\author{
P. N. JOHNSON-LAIRD \\ University of Sussex, Brighton, England
}

The question is prompted by Eugene Charniak's (1978) recent editorial introducing the new 'Theoretical Notes' section. The idea of such a section is excellent, but I am dubious about one aspect of it. Charniak writes that one of its functions will indeed be to present neat solutions to small problems. He illustrates the idea with R. McGuire's observation of the difference in the acceptability of the following sentences as ways of describing the normal mode of entering vehicles:

Fred got on the train.

*Fred got in the train.

Fred got in the car.

*Fred got on the car.

The difference, according to McGuire, reflects the fact that one only uses 'get on' when one can stand up in the thing, 'get in' when one can't. This solution is neat, but wrong.

The problems arise if one considers other cases. For example, the sentence:

Fred got on the stool

is acceptable and may mean that he sat on it in the usual way. Of course, you can stand on a stool, which correctly predicts:

*Fred got in the stool

as a way of describing him sitting on it. However, one can also stand on a chair, and yet if Fred is a baby or a very small person, then both:

Fred got on the chair (e.g. he had to climb up onto it) and:

Fred got in the chair (e.g. he sat well back with his legs tucked up under him) 
are acceptable. There is a similar phenomenon in the case of:

Fred got on the bed (e.g. he lay down on top of the covers) and:

Fred got in the bed (e.g. he got beneath the covers)

and you can stand on a bed-even with all the bedcovers over you, as my children often demonstrate. Evidently, getting on in life is not merely a matter of standing up to be counted.

Turning to the other half of McGuire's thesis, there are plenty of cases where getting in is acceptable even though standing up is not only possible but actually the norm:

Fred got in the elevator

Fred got in the gondola of the balloon

Fred got in the tube at Piccadilly Circus.

According to McGuire, one ought to say, for example:

Fred got on the elevator

which is odd, and suggests that he clambered through the hatch to get on top of it. Moreover, if one tries to generalize McGuire's solution to other verbs, still worse befalls: an exercise that I leave to the reader.

What is really going on (or in)? The answer, I believe, is to be found by extending the analyses of Miller and Johnson-Laird (1976, Sec. 6.1.1). The relevant sense of $x$ is in $y$ can be paraphrased as at least part of $x$ is spatially included within $y$, e.g.:

$$
\text { Fred is in }\left\{\begin{array}{l}
\text { the car } \\
\text { the chair } \\
\text { the bed } \\
\text { the elevator } \\
\text { the gondola of the balloon } \\
\text { the tube. }
\end{array}\right.
$$

The only way in which you make sense of:

*Fred is in the stool

is by imagining either that he has somehow inserted himself between its legs or else that he is a Lilliputian who has got in some hollow part of it. The case of $x$ is on $y$ is more complicated, because it appears to have an ambiguity that is relevant to the analysis. One sense of the relation can be paraphrased roughly as $x$ is supported by an upper surface of $y$, e.g.: 
Fred is on $\left\{\begin{array}{l}\text { the car } \\ \text { the stool } \\ \text { the chair } \\ \text { the bed } \\ \text { the elevator }\end{array}\right.$

Another sense of $x$ is on $y$ can be paraphrased as $x$ is located within $y$ where $y$ is conceived of as a surface, e.g.:

$$
\text { Fred is on }\left\{\begin{array}{l}
\text { the ship } \\
\text { the river } \\
\text { the island. }
\end{array}\right.
$$

Whether there is a genuine ambiguity here, or merely a sort of referential indeterminacy is perhaps open to doubt. However, certain sentences seem open to both interpretations:

Fred is on $\left\{\begin{array}{l}\text { the train } \\ \text { the tube } \\ \text { the gondola. }\end{array}\right.$

Likewise, conjunction reduction, one of Zwicky and Sadock's (1973) diagnostic tests for identity of sense, seems odd when the two interpretations are combined, e.g.:

Fred got on the ship and the table.

This last example uses the verb to get, which can be paraphrased as $x$ does something that causes $x$ to be in a certain state, and accordingly $x$ gets in $y$ means that $x$ causes $x$ to be in $y$, whereas $x$ gets on $y$ means $x$ causes $x$ to be on $y$. It follows according to this analysis that:

Fred got on the train

is an acceptable but ambiguous sentence: he may have boarded the train in the usual way or, rather more unconventionally, he may have got on its roof. Likewise, the sentence:

Fred got in the train

is acceptable (pace McGuire) and describes the act of boarding it; the usage is more acceptable in England, where platforms exist, than in America, where the notion of climbing up into the train suggests getting up onto it. The sentence:

Fred got in the car is an acceptable way of describing the usual way of entering a vehicle. Finally:

Fred got on the car 
means that he got on its roof, because cars are too small to be treated as conceptual surfaces.

This account is hardly the last word on the semantics of prepositions, but at least it allows us to draw a moral: what seemed like a neat solution was so only because the problem was small. In trying to find a more adequate solution, one is forced willy-nilly to consider an ever wider range of phenomena, the problem soon ceases to be small. To account for a simple verb and preposition, one is forced to consider other verbs and other prepositions, tests for ambiguity as opposed to indeterminacy, and the conception of three-dimensional objects as surfaces. This growth in complexity seems a characteristic of considering any aspect of human mentality. There may not be many small problems in cognitive science, or any neat solutions to them in Cognitive Science.

\section{ACKNOWLEDGMENTS}

I am grateful to Kate Ehrlich for a critical reading of this note, and to the S.S.R.C. (GB) for research support.

\section{REFERENCES}

Chamiak, E. Editorial. Cognitive Science, 1978, 2, 193-194.

Miller, G. A., \& Johnson-Laird, P. N. Language and perception. Cambridge, Mass.: Harvard University Press. London: Cambridge University Press, 1976.

Zwicky, A. M., \& Sadock, J. M. Ambiguity tests and how to fail them. Working Papers in Linguistics, Ohio State University, No. 16, 1-34, 1973. 